\title{
A retrospective Evaluation of Antibacterials Used in the Management of Diabetics in a Tertiary Hospital in South-western Nigeria
}

Mopelola Ibidunni Ayeni ${ }^{1}$, Ezekiel Olugbenga Akinkunmi ${ }^{2}$, RosemaryTemitope Ikem $^{3}$, Patrick Osemene ${ }^{1}$ and Adunni Morohunfola $^{4}$

1. Department of Clinical Pharmacy and Pharmacy Administration. Obafemi Awolowo University, Ile-Ife, Osun State.22005.Nigeria

2. Department of Pharmaceutics, Obafemi Awolowo University, Ile-Ife, Osun State 22005.Nigeria

3. Department of Medicine, Obafemi Awolowo University, Ile-Ife, Osun State 22005.Nigeria

4. Tots \& Tykes Pediatrics, 3750 S University Dr Ste 200 Fort Worth, TX 76006. USA

\begin{abstract}
Objective: Co-medication with antibacterials is often necessary in the management of diabetic patients. There is a constant need to evaluate the use of these antibacterials to improve therapeutic outcomes and to avoid practices that could possibly increase the emergence of antimicrobial resistance. The aim of this study was to evaluate the utilization of antibacterials in the diabetic clinic of a Nigerian tertiary hospital. Methods: A retrospective evaluation and analysis of patients' case notes written by physicians in the diabetic clinic over a three-year period (January, 2008-December, 2010) was conducted. The case notes were the individual medical records for 150 different hospitalized diabetic patients with infection as co-indication. Data was collected using a WHO instrument for studies of rational drug use. Results: Descriptive statistics such as mean, frequency, percentages and standard deviation were used to analyze categorical data obtained from the demographic variables. Fisher exact test and chi square test were employed to determine whether there were statistically significant differences or associations between mean values of antibacterial prescription pattern and attendant dosage errors. ( $\mathrm{P}=1<0.05$ was accepted as significant). Comparing the mean number of antibacterial drugs in each prescription yearly yielded a mean of 1.32 in 2008, 1.37 in 2009 and 1.67 in 2010, indicating that there was no statistically significant difference. Antibacterials were used to manage complications often seen in the diabetic patient, including; diabetic foot gangrene, injection abscess, septiceamia, diabetic foot ulcer, vaginal yeast infection, amongst others. Conclusions: The majority of prescriptions met the criteria for compliance with the hospital's drug use protocol. The few instances of non-compliance with the hospital's antibiotic drug use protocol may indicate the need for continuing education, especially for the less experienced prescribers.
\end{abstract}

Key words: Antibiotics, diabetes, infection, WHO, Nigeria.

\section{Introduction}

Diabetes mellitus is a chronic metabolic disorder found in African communities and is also a significant cause of morbidity and mortality worldwide [1]. It has been suggested that on average, a patient with diabetes spends approximately 6 days a year in the hospital compared with an average of 1 day for a non-diabetic patient [2]. In contrast to coronary artery disease and

Crresponding author: Mopelola Ibidunni Ayeni, Ph.D. student, research field: pharmacoepidemiology and clinical pharmacokinetics. E-mail: mopeayeni@yahoo.com and mayeni@oauife.edu.ng. cerebrovascular disease which are the reported main causes of diabetic death in the developed world, in the developing countries leading causes of death include infections and acute metabolic complications [3-5].

Antibacterial drugs are recommended for the treatment of infections associated with diabetes [6, 7]. One important challenge for physicians involved in the care of diabetics is the choice of appropriate antibacterial agents. The challenge is made more complex by the so-called new emerging (infectious) diseases and resistance of bacterial pathogens to traditional antibacterial drug treatment [8, 9]. In 
addition, just like all other drugs, antibacterials have side-effects and can have drug-drug interactions with other concomitantly used drugs, including other antibacterials. Also, it has been recommended that some antibiotics, such as gatifloxacin and levofloxacin, should be used with caution in diabetic patients [10]. Hence, physicians involved in treating diabetic patients need to be cautious and continuously update their knowledge about the use of antibacterials, in conjunction with specific anti-hyperglycemic drugs.

Adequate appreciation of the characteristics of antibacterial drugs will lead to their rational prescribing and optimal benefit for the patients [11, 12]. This study was designed to investigate prescription patterns using WHO standardized drug use indicators. It involves investigation of the compliance with the standards/guidelines concerning the rational use of antibacterials, including antibiotics, the correctness of doses, possible adverse drug reactions, drug-drug interactions, and making recommendations to prescribers.

\section{Materials and Methods}

The medical records of all admissions into the medical wards of the Obafemi Awolowo University Teaching Hospitals Complex (OAUTHC), Ile-Ife in the South Western part of Nigeria, from January 2008 to December 2010 were retrieved and studied. OAUTHC is a tertiary health institution serving over 5 million people consisting of both in-patients and out-patients from all over Nigeria. Permission to access data was given by the institutional review board of the hospital and ethical approval was obtained. All cases notes with the diagnosis of diabetes complicated with infections were selected making a total of 150 individual patients with such cases within the study period. Data collected from the case notes included relevant demography data (such as age and sex), diabetic complications, type, dose, frequency and duration of antibacterial drugs prescribed, concurrently prescribed drugs, and dosing instructions. The study was designed to identify deviations from drug use protocol of the institution. The protocol suggested that antibacterial drugs used in the hospital shall satisfy the following criteria:

- Improve patient care by promoting the best practice in proper dosing, antibiotic prophylaxis and therapy

- Make better use of resources by using cheaper generics drugs where possible

- Delay the emergence and spread of multiple antibiotic-resistant bacteria

- Eliminate the use of unnecessary or ineffective antibacterial agents and restrict the use of expensive or unnecessarily powerful ones so as to save them for more complicated and resistant infections.

The study examined deviations from the hospital protocol including indications for prophylaxis and treatment of infection, the optimum dosages, timings and duration of therapy and ability to identify drug incompatability, toxicity and adverse drug reactions. Data were only extracted from the patient's case notes, and no interviews were conducted. For the purpose of this study, indicator studies were restricted to a sample of patients suffering mainly to complications of adult onset diabetes. A standardized drug-use core indicators questionnaire designed and tested by World Health Organization was used (WHO, 1993). A number of indicators have been developed, standardized and evaluated by World Health Organization (WHO). These indicators are grouped in to three categories namely: prescribing indicators, patient care indicators and facility indicators [13]. For the purpose of this study, only indicators of the first category were used. Data was collected by using WHO designed criteria based data collection formats for retrospective study. According to WHO drug use evaluation guideline, prescribing indicators used include; average number of drugs per encounter, percentage of drugs prescribed in generics, percentage of prescriptions with antibiotics, percentage of prescriptions with injections and percentage of prescribed drugs from EDL (essential 
drug list). Descriptive statistics such as mean, frequency, percentages and standard deviation were used to analyze categorical data obtained from the demographic variables. Fisher exact test and chi square test were employed to determine whether there were statistically significant differences or associations between mean values of antibacterial drugs prescription pattern and attendant dosage errors. ( $\mathrm{P} \leq$ 0.05 was accepted as significant).

\section{Results}

The indications for the use of antibacterials in the management of the diabetic patients at the clinic included diabetes complications with infections or associated infectious conditions which included diabetic foot gangrene, injection abscess, septicaemia, vaginal infections, diabetic foot ulcer, decubitus ulcer, septic arthritis, discharging leg sinus, acute abdomen, tuberculosis, skin infections, meningitis, multiple diabetic ulcer, scrotal ulcer, gastroenteritis, and glomerulonephritis. The majority of the cases relates to patients with adult onset diabetes, with a two year old patient as the only exception. Prescriptions in one hundred and fifty case notes were reviewed. In all case notes the gender, age and diabetes complications of the patients were indicated. Of all patients, 95 (63.3\%) were males and $55(36.7 \%)$ were females. The mean age was found to be $56.5 \pm 14.1$ (range two to eighty years). Over the three years, the mean number of the antibacterial drugs used was $1.39 \pm 0.7$ (zero to three) with a mode of one. Antibacterial drugs were included in $133(88.7 \%)$ of the 150 case notes. The key prescribing indicators are stated in Table 1 . When the mean number of antibacterial drugs per case note per year was compared at P-Value of 0.05 , the result showed a mean of 1.32 in 2008, 1.37 in 2009 and 1.67 in 2010, showing no statistically significant difference with a standard deviation value of 0.86 for 2008/2009 and 0.78 for 2009/2010. Antibacterial drugs were included in $133(88.7 \%)$ of the 150 prescriptions evaluated. As shown in Table 2, metronidazole was prescribed in $52(34.7 \%)$ of the 150 prescriptions, accounting for the antibacterial agent with the highest rate of prescription for diabetics at the centre. The rate of metronidazole prescription increased from an initial $30 \%$ in 2008 to $43.9 \%$ in 2009 and then decreased to

Table 1 Prescribing indicators.

\begin{tabular}{lllll}
\hline Serial No & Year & Average antibacterial drugprescribed per prescription & Generics $(\%)$ & Parenteral $(\%)$ \\
\hline 1 & 2008 & 1.32 & 78.0 & 48.0 \\
2 & 2009 & 1.37 & 50.0 & 52.3 \\
3 & 2010 & 1.67 & 67.2 & 36.0 \\
\hline
\end{tabular}

Table 2 Distribution of antibacterial drug prescriptions and dosage errors.

\begin{tabular}{|c|c|c|c|c|c|c|c|c|}
\hline Drug & $\begin{array}{l}2008, \text { Number } \\
\text { (\% of 50Rx) }\end{array}$ & $\begin{array}{l}\text { Dosage } \\
\text { errors }\end{array}$ & $\begin{array}{l}2009 \text { No }(\% \\
\text { of } 41 \mathrm{Rx})\end{array}$ & $\begin{array}{l}\begin{array}{l}\text { Dosage } \\
\text { errors }\end{array} \\
\end{array}$ & $\begin{array}{l}2010 \mathrm{No}(\% \\
\text { of 59Rx) }\end{array}$ & $\begin{array}{l}\text { Dosage } \\
\text { errors }\end{array}$ & $\begin{array}{l}2008-2010, \text { No } \\
(\% \text { of } 150 \mathrm{Rx})\end{array}$ & $\begin{array}{l}\text { Dosage } \\
\text { errors }\end{array}$ \\
\hline $\begin{array}{l}\text { Metronidazole } \\
\left(\text { Flagyl }^{\mathrm{R}} \text { ) }\right.\end{array}$ & $15(30)$ & 0 & $18(43.9)$ & 2 & $19(32.2)$ & 2 & $52(34.7 \%)$ & 4 \\
\hline Ciprofloxacin & $21(42)$ & 2 & $15(36.6)$ & 1 & $13(22.0)$ & 1 & $49(32.7 \%)$ & 4 \\
\hline Amoxycillin & $8(16)$ & 0 & $11(26.9)$ & 1 & $11(18.6)$ & 1 & $30(20.0 \%)$ & 2 \\
\hline Erythromycin & $7(14)$ & 0 & $1(2.4)$ & 1 & $15(25.4)$ & 1 & $23(15.3 \%)$ & 2 \\
\hline Ceftriaxone & $4(8)$ & 0 & $5(12.2)$ & 0 & $13(22.0)$ & 0 & $22(14.7 \%)$ & 0 \\
\hline Cefuroxime & $2(4)$ & 0 & $2(4.8)$ & 0 & $4(6.8)$ & 0 & $8(5.3 \%)$ & 0 \\
\hline Augmentin $^{R}$ & $5(10)$ & 0 & 0 & 0 & $1(1.7)$ & 0 & $6(4.0 \%)$ & 0 \\
\hline Ampiclox ${ }^{R}$ & $1(2)$ & 0 & $1(2.4)$ & 0 & $2(3.4)$ & 0 & $4(2.7 \%)$ & 0 \\
\hline Nystatin & $2(4)$ & 0 & 0 & 0 & $2(3.4)$ & 0 & $4(2.7 \%)$ & 0 \\
\hline Azithromycin & $1(2)$ & 0 & $1(2.4)$ & 0 & $1(1.7)$ & 0 & $3(2.0 \%)$ & 0 \\
\hline Chloramphenicol & $1(2)$ & 0 & 0 & 0 & $1(1.7)$ & 0 & $2(1.3 \%)$ & 0 \\
\hline Total & 67 & 2 & 54 & 5 & 82 & 5 & 203 & 12 \\
\hline
\end{tabular}




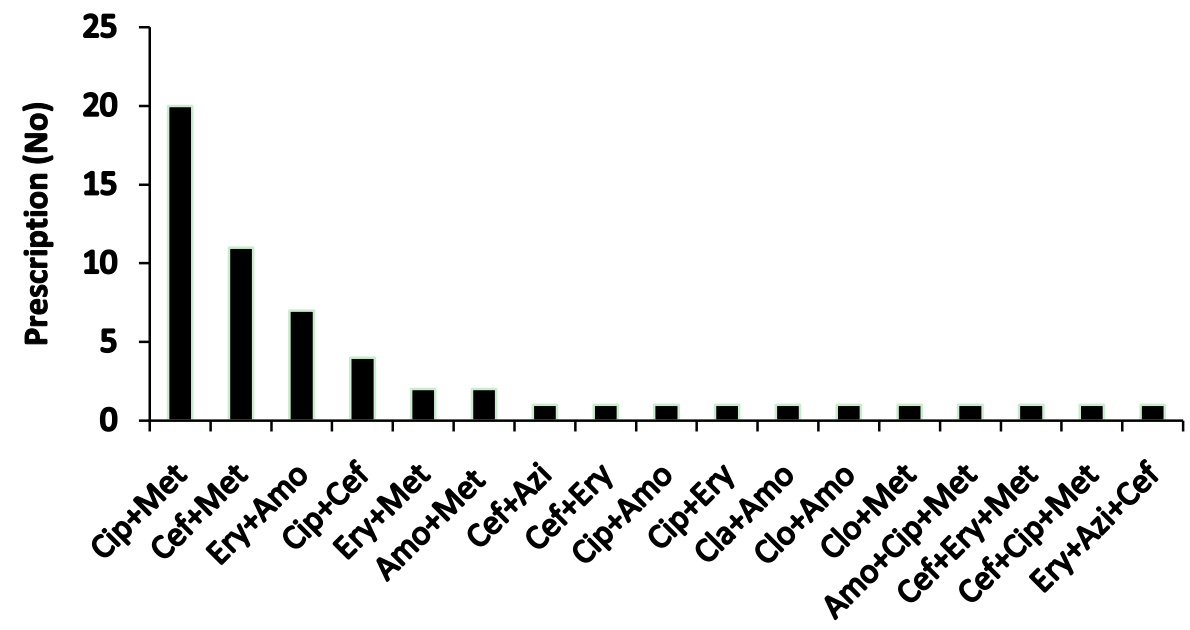

Antibiotics

Fig. 1 Multiple antibiotic prescription in the diabetics.

Met: Metronidazole; Clp: Ciprofloxacin; Amo: Amoxycillin; Ery: Erythromycin;

Cef: Ceftriazone; Azi: Azithromycin; Clo: Cloxacillin; Cla: Clarithromycin; +: Co-prescription

$32.2 \%$ in 2010. Ciprofloxacin was next to metronidazole in frequency of prescription and was prescribed in 49 (32.7\%) of the 150 cases. The rate of prescription of ciprofloxacin decreased through the years. Amoxycillin was used in $30(20.0 \%)$ cases, erythromycin in $23(15.3 \%)$, ceftriaxone in $22(14.7 \%)$, cefuroxime in $8(5.3 \%)$, augmentin ${ }^{\mathrm{R}}$ in $6(4.0 \%)$, ampiclox ${ }^{\mathrm{R}}$ and nystatin in $4(2.7 \%)$, azithromycin in 3 (2.0\%), and chloramphenicol in only 2 cases as eye drops in the three years. Other antibacterials used included; ofloxacin, cloxacillin, clarithromycin, Septrin $^{\mathrm{R}}$ and nitrofurantoin which were used at a rate of less than $1.0 \%$ each.

The combination of ciprofloxacin with metronidazole was prescribed in $20(13.3 \%)$ of the (150) cases. Metronidazole was also prescribed with ceftriaxone, amoxicillin and erythromycin in $11(7.3 \%)$, $2(1.3 \%)$ and $2(1.3 \%)$ cases respectively. In 7 (4.7\%) of the cases amoxycillin and erythromycin were prescribed together. In 4 different cases a combination of 3 antibacterials was prescribed, namely: ceftriaxone/ erythromycin/ azithromycin; ceftriaxone/ erythromycin/metronidazole; ciprofloxacin/ metronidazole; and amoxicillin/ ciprofloxacin/ metronidazole (Fig. 1).
Over the three years, the dosages for $4(7.7 \%)$ of the 52 metronidazole, $4(8.2 \%)$ of the 49 ciprofloxacin, $2(6.7 \%)$ of the 30 amoxicillin and $2(8.7 \%)$ of the 23 erythromycin prescriptions were incorrectly written. All the prescriptions for the remaining antibacterial agents had the correct dosages.

\section{Discussion}

It was found in this study that the mean age of the patients was $56.46 \pm 14.1$. This group of almost exclusively adult patients does not form a homogenous group, therefore there are notable differences in both the individual handling of and the response to the drugs prescribed, including the antibiotics and other antibacterials [14]. Therefore the general rule of prescribing the minimum number of drugs should be borne in mind by the prescribers and every drug prescribed should have a strong indication for its use.

This study revealed that the appropriate drugs were mostly selected from the hospital formulary and the 5 most frequently prescribed antibacterials (singly or in combination) could be identified. Metronidazole was the most frequently prescribed antibacterial (34.7\%), followed by ciprofloxacin (32.7\%), amoxicillin (20.0\%), erythromycin (15.3\%) and ceftraxone 
(14.7\%).

Metronidazole is one of the drugs of choice in anaerobic infections and most diabetic infections are anaerobic $[15,16]$. It is not surprising therefore that this drug was prescribed with the highest frequency at the hospital during the period under investigation.

Ciprofloxacin, apart from a single prescription of ofloxacin, was the only quinolone prescribed during the three years under investigation. It is also noted that the rate of prescription of ciprofloxacin decreased through the years. This is in agreement with the problems reported to be associated with the use of the quinolones in diabetic patients. It has been reported that the use of levofloxacin in diabetics was associated with a slightly increased risk of hypoglycaemia while gatifloxacin use was associated with both hypoglycaemia and hyperglycaemia [17]. In addition, the frequency of dysglycemia occurring after antibiotic use has been reported to be highest for the quinolones but least for the second-generation cephalosporins and macrolides [10]. This could be the reason why erythromycin, and the new generation cephalosporin, ceftriaxone and cefuroxime were prescribed in an increasing rate through the years under study.

Moreover, ciprofloxacin is also known to be associated with tendinopathy, tendon rupture and hypotension which are problems of importance in the elderly [18]. An awareness of these problems by the prescribers was mainly responsible for the continual reduction in the use of this drug in the hospital during the years under investigation.

In this study, co-prescriptions of antibacterial drugs were observed in some cases. These combinations are rational and they should give enhanced activity against both aerobic and anaerobic pathogens. For instance, ceftriaxone and erythromycin are antibiotics indicated for the treatment of infections caused by organisms resistant to the penicillins, therefore their co-prescription with amoxicillin and other antibiotics as observed in certain cases in this study is expected to be synergistic. No antibiotic-antibiotic incompatibility was detected in this study.

Some discrepancies such as omission of dosages and/or duration of drug usage were noted in $5.9 \%$ of the prescriptions. Appropriate and correct use of antibacterial drugs has been advised to ensure that effective and safe treatment is available and practices that may enhance antimicrobial resistance are avoided [14]. Dosages should conform to accepted pharmacokinetic standard and take into consideration the patient's age, as well as, the presence of kidney and liver disease which is more prevalent in the elderly than patients of other age groups [14].

Furthermore it was observed that a higher rate of generics was prescribed compared with branded drugs. Drug prescribers are often encouraged to prescribe generics mostly for economic reasons. This forms part of the essential drug practices in the hospital [19]. It was discovered that the $28 \%$ fall in the prescription of generics in the year 2009 (50\%) when compared to year 2008 (78\%) was due to a transient supply shortage.

It was observed in this study that the parenteral route of drug administration was employed at a lower rate than the oral route. The choice of the route of administration of drugs depends a lot on the property of the drugs and the condition of the patients. The variations in the choice of the route of drug administration over the years were therefore not unexpected, considering the fact that parenteral routes are known to increase the risk of infection abscess in diabetic patients.

\section{Conclusion}

The study concludes that antibacterials are widely used in the diabetic clinic of the hospital for the management of diabetics that are co-indicated with infections. The majority of prescriptions of antibacterial drugs met the criteria for compliance. The few instances of non-compliance with the hospital's antibiotic drug use protocol may indicate the need for continuing education especially for the less 
experienced prescribers. A training program should be developed to improve adherence to prescribing guidelines. .

\section{References}

[1] Mufunda, J., Chatora, R., Ndambakuwa, Y., Nyarango, P., Kosia, A., Chifamba, J., Filipe, A., Usman, A., and Sparks, V. H. 2008. "Emerging non-communicable disease epidemic in Africa: preventive measures from the WHO Regional Office for Africa.” Ethnic Dis. (16) 512-6.

[2] Wagner, E. H., Sandhu, N., and Newton, K. 2001. "Effect of Improved Glycaemic Control on Health Care Costs and Utilisation." JAMA 285:182-9.

[3] Janka, H. U. 1996. "Increased Cardiovascular Morbidity and Mortality in Diabetes Mellitus: Identification of the High Risk Patients." Diabet Res. Clin. Prac. (30) 85-8.

[4] Zargar, A. H., Wani, A. I., Masoodi, S. R., Laway, B. A., and Bashir, M. I. 1999. "Mortality in Diabetes Mellitus Data from a Developing Region of the World." Diabet Res. ClinPrac. (43) 67-74.

[5] Adekanle, O., Ayodeji, O. O., Olatunde, L. O., and Folorunso, T. R. 2008. "A 7-Year Retrospective Study of Diabetes Related Deaths in a Nigerian Tertiary Hospital." Diabetes International 11: 15-17.

[6] Lipsky, B. A. 2010. "New Developments in Diagnosing and Treating Diabetic Foot Infections." Diab Met. Res. Rev. 28 (Suppl 1): 566-71.

[7] Leese, G. P. 2009. "Review: The varied attractions of the diabetic foot." British Journal of Diabetes \& Vascular Disease (9): 155-9.

[8] Orji, F. A., Nwachukwu, N. C., and Udora, E. C. 2009. "Bacteriological Evaluation of Diabetic ulcers in Nigeria." African Journal of Diabetes Medicine 17(2) 19-21

[9] Unachukwu, C. N., Obunge, O. K., and Odia, O. J. "The Bacteriology of diabetic foot ulcers in Port Harcourt."
NOiger J Med 14 (2):173-6.

[10] Kendall, C., and Wooltorton, E. 2008. "People with Diabetes should Avoid Antibiotic Gatifloxacin." Canadian Medical Association Journal 174 (8): 1089

[11] Bakssas, I., and Lunde, P. K. M. 1986. "National Drug Policies: the Need for Drug Utilization Studies." Trends Pharmacol Sci 7: 331-4.

[12] Ogunbodede, E. O, Fatusi, A. O., Folayan, M. O., and Olayiwola, G. 2005. "Retrospective Survey of Antibiotic Prescriptions in Dentistry." J. Contemp. Dent. Pract. 6 (2) 64-71.

[13] WHO. 1993. How to Investigate Drug Use in Health Facilities: Selected Drug Use Indicators. Geneva, World Health Organization. WHO/DAP 1: 1-87.

[14] Matzke, G. R., and Kovarik, J M. 1992. Clinical pharmacokinetics of anti-infectives in: Applied Therapeutics - The Clinical Use of Drugs. 5th Edition Pub: Applied Therapeutics, Inc. 34 (1992) 1-16.

[15] Steinke, D. T., Seaton, R. A., Phillips, G., MacDonald, T. M., and Davey, P. G. 1999. "Factors Associated with Trimethoprim-resistant Bacteria Isolated from Urine Samples." Journal of Antimicrobial Chemotherapy 43: 841-3.

[16] Bansal, E., Garg, A., Bhatia, S., Altri, A. K., and Chander, J. 2010. "Spectrum of Microbial Flora in Diabetic Foot Ulcers." Indian J. Med. Microbiol. 51: 204-8.

[17] Park-Wyllie, L. Y., Juurlink, D. N., Kopp, A., Shah, B. R., Stukel, T. A., Stumpo, C., Dresser, L., Low, D. E., and Mamdani, M. M. 2006. "Outpatient gatifloxacin therapy and dysglycemia in older adults." N. Engl. J. Med. 354 (13): 1352-61.

[18] Sweetman, S. C, ed. 2005. Martindale. The Complete Drug Reference. London: Pharmaceutical Press. 238-9.

[19] Federal Republic of Nigeria. Essential Drug List, Third Revision. The Federal Ministry of Health, Lagos, Nigeria, 1996. 